\title{
Correction to: Haantjes algebras of classical integrable systems
}

\author{
Piergiulio Tempesta $^{1,2}$ (D) Giorgio Tondo ${ }^{3}$
}

Accepted: 9 October 2021 / Published online: 25 October 2021

(c) Fondazione Annali di Matematica Pura ed Applicata and Springer-Verlag GmbH Germany, part of Springer

Nature 2021

\section{Correction to: Annali di Matematica Pura ed Applicata (1923 -) https://doi.org/10.1007/s10231-021-01107-4}

The original version of this article was published on 28th May, 2021. In the final, published version, the following changes have been made in page 6 and 30 of the article.

The line in page 6 that previously read as "The assumptions (8), (9) ensure that the set $\mathrm{H}$ is an associative algebra of Haantjes operators; moreover, the Hamilton-Cayley theorem implies that its rank $\mathrm{m}$ is not greater than $\mathrm{n} . "$

Should read as "The assumptions (8), (9) ensure that the set $\mathscr{H}$ is an associative algebra of Haantjes operators."

The line in page 30 that previously read as "still a basis of a Haantjes algebra. However, this algebra is non-Abelian; therefore, it does not admit a generator. Thus, the PW system is a remarkable example of a superintegrable system admitting a non-Abelian $\omega \mathrm{H}$ structure of rank 3."

Should read as "a set of generators of a Haantjes algebra. However, this algebra is nonAbelian; therefore, it does not admit a generator. Thus, the PW system is a remarkable example of a superintegrable system admitting a non-Abelian $\omega \mathscr{H}$ structure of rank 4."

The original article has been corrected.

Publisher's Note Springer Nature remains neutral with regard to jurisdictional claims in published maps and institutional affiliations.

The original article can be found online at https://doi.org/10.1007/s10231-021-01107-4.

Piergiulio Tempesta

p.tempesta@fis.ucm.es; piergiulio.tempesta@icmat.es

Giorgio Tondo

tondo@units.it

1 Departamento de Física Teórica II, Facultad de Físicas, Universidad Complutense, 28040 Madrid, Spain

2 Instituto de Ciencias Matemáticas, C/ Nicolás Cabrera, No 13-15, 28049 Madrid, Spain

3 Dipartimento di Matematica e Geoscienze, Università degli Studi di Trieste, Piaz.le Europa 1, 34127 Trieste, Italy 